\title{
Solitons in a chain of parity-time-invariant dimers
}

\author{
Sergey V. Suchkov, ${ }^{1}$ Boris A. Malomed, ${ }^{2,3}$ Sergey V. Dmitriev, ${ }^{1}$ and Yuri S. Kivshar ${ }^{4}$ \\ ${ }^{1}$ Institute for Metals Superplasticity Problems, Russian Academy of Science, Ufa 450001, Russia \\ ${ }^{2}$ Department of Physical Electronics, School of Electrical Engineering, Faculty of Engineering, Tel Aviv University, Tel Aviv 69978, Israel \\ ${ }^{3}$ Institut de Ciencies Fotoniques, Mediterranean Technology Park, Castelldefels (Barcelona) 08860, Spain \\ ${ }^{4}$ Nonlinear Physics Center, Research School of Physics and Engineering, Australian National University, Canberra ACT 0200, Australia
}

(Received 28 April 2011; revised manuscript received 28 July 2011; published 21 October 2011)

\begin{abstract}
Dynamics of a chain of interacting parity-time-invariant nonlinear dimers is investigated. A dimer is built as a pair of coupled elements with equal gain and loss. A relation between stationary soliton solutions of the model and solitons of the discrete nonlinear Schrödinger (DNLS) equation is demonstrated. Approximate solutions for solitons whose width is large in comparison to the lattice spacing are derived, using a continuum counterpart of the discrete equations. These solitons are mobile, featuring nearly elastic collisions. Stationary solutions for narrow solitons, which are immobile due to the pinning by the effective Peierls-Nabarro potential, are constructed numerically, starting from the anticontinuum limit. The solitons with the amplitude exceeding a certain critical value suffer an instability leading to blowup, which is a specific feature of the nonlinear parity-time-symmetric chain, making it dynamically different from DNLS lattices. A qualitative explanation of this feature is proposed. The instability threshold drops with the increase of the gain-loss coefficient, but it does not depend on the lattice coupling constant, nor on the soliton's velocity.
\end{abstract}

DOI: 10.1103/PhysRevE.84.046609

PACS number(s): 05.45.Yv, 42.25.Bs, 11.30.Er, 42.82.Et

\section{INTRODUCTION}

In the original works by Bender et al. [1,2], it was pointed out that non-Hermitian Hamiltonians can have an entirely real eigenvalue spectrum under the parity-time $(\mathcal{P} \mathcal{T})$-symmetry constraint. This mathematical observation can have deep physical consequences, essentially altering the familiar properties of collective modes in the respective media combining amplification and dissipation. Indeed, it is usually assumed that the balance between the gain and loss uniquely selects parameters of isolated stable modes. However, the use of the $\mathcal{P} \mathcal{T}$ symmetry makes it possible to support continuous families of modes, allowing the dissipative media to emulate conservative ones, up to a certain threshold level. In particular, the effects of amplification and dissipation may stay in the exact balance for symmetric configurations of weak fields; hence the $\mathcal{P} \mathcal{T}$-invariant dynamics is preserved. In contrast, for field intensities above the threshold, nonlinear self-action (if present in the system) breaks the $\mathcal{P} \mathcal{T}$ symmetry both locally and globally, resulting in the asymmetric wave localization in the region with amplification.

As the necessary condition for the $\mathcal{P} \mathcal{T}$ symmetry of the Hamiltonian with a complex potential, $V(x)$, is reduced to condition $V(x)=V^{*}(-x)$, such systems can be realized in the most straightforward way in optics, by combining a spatially symmetric profile of the refractive index with symmetrically placed mutually balanced gain and loss [4] (complex potentials may also be physically relevant in the case when they are not subject to the $\mathcal{P} \mathcal{T}$-symmetry constraint [3]). The possibility to realize physical systems with the $\mathcal{P} \mathcal{T}$ symmetry was a motivation for many theoretical [5] and experimental [6] works. Effects of the conservative nonlinearity in $\mathcal{P} \mathcal{T}$-symmetric systems were addressed too [7-9]. Recently, the nonlinearity of the gain and loss, also subject to the condition of the $\mathcal{P} \mathcal{T}$ symmetry, was introduced in Ref. [10].

The simplest nonlinear $\mathcal{P} \mathcal{T}$-symmetric object can be realized as a pair of linearly coupled optical waveguides (also called a dimer), composed of a passive waveguide carrying linear loss and its active counterpart imparted with a matched compensating gain [8] (in a more general form, without the condition of the exact equilibrium between the loss and gain, the same system of linearly coupled active and passive waveguiding cores was investigated for a long time in various forms [11-15]; see also a review in Ref. [16]). A chain of such $\mathcal{P} \mathcal{T}$-invariant couplers was studied in Ref. [9], with each active or passive element linearly connected to an element of the opposite sign, belonging to an adjacent coupler. In other words, in this setting the axis of each waveguiding coupler is perpendicular to the direction along which the chain is built. It was demonstrated that this chain of couplers can support stable solitons with amplitudes smaller than a threshold amplitude.

In the present work, we propose and analyze another array of $\mathcal{P} \mathcal{T}$-symmetric couplers with the intrinsic nonlinearity. In the array, active and passive elements are linearly coupled to the elements of the same sign belonging to adjacent dimers [see Fig. 1(a)]; i.e., the axes of the couplers are aligned with the direction of the chain composed of them. In fact, the proposed system is quite natural in terms of the realization in optics, as it can be built of two parallel extended waveguides, one pumped and one lossy, if each one is segmented into an array of individual waveguides. Our aim is to find nonlinear localized modes (discrete solitons) in this chain, and study their stability, mobility, and interaction.

The paper is organized as follows. Section II outlines the model. Analytical results for moving and stationary solitons and their stability are presented in Sec. III. Results of numerical studies are reported in Sec. IV. Section V concludes the paper.

\section{MODEL}

As said above, we consider a nonlinear chain, shown in Fig. 1(a), which is described by the following set 


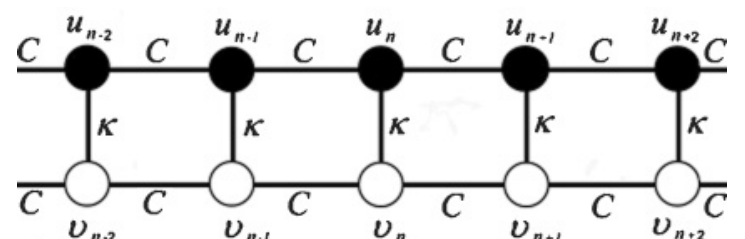

(a)
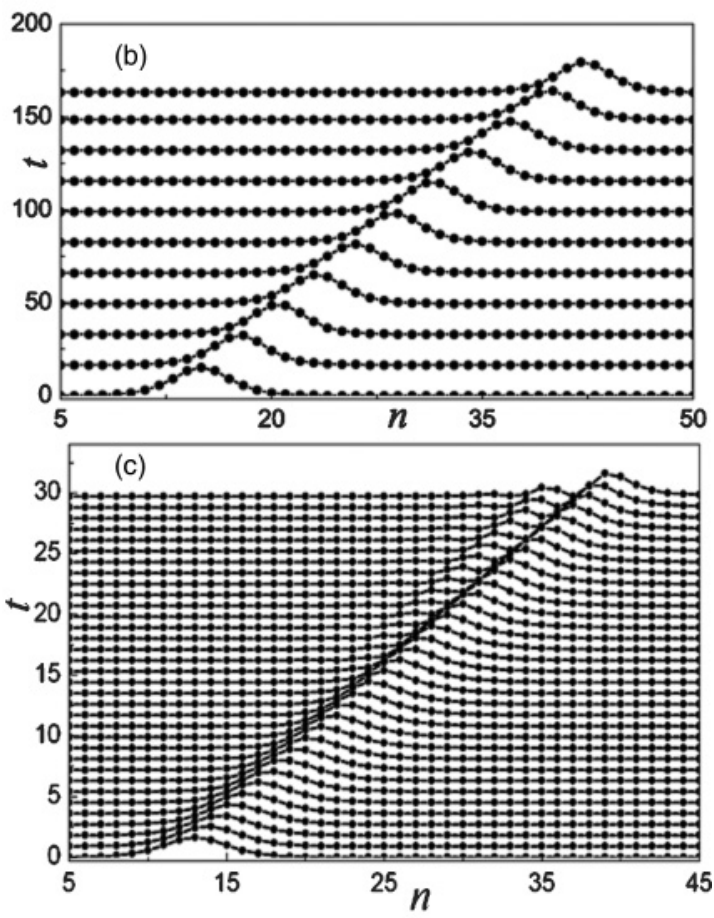

FIG. 1. (a) Schematic of the chain of dimers. (b) Spatiotemporal evolution of $\left|u_{n}(t)\right|^{2}$ showing the propagation of a soliton through the chain of dimers described by Eq. (2) [the respective plot for $\left|v_{n}(t)\right|^{2}$ is nearly identical to this one]. The initial conditions were set as per the approximate solution (6), with $\gamma=0.5, A=0.37, C=0.5$, and $V=0.2$. (c) Same as in (b), but for a larger velocity, $V=2$.

of equations:

$$
\begin{aligned}
\frac{d u_{n}}{d t}= & +\gamma u_{n}+i \sigma\left|u_{n}\right|^{2} u_{n}+i \kappa v_{n} \\
& +i C\left(u_{n+1}+u_{n-1}-2 u_{n}\right), \\
\frac{d v_{n}}{d t}= & -\gamma v_{n}+i \sigma\left|v_{n}\right|^{2} v_{n}+i \kappa u_{n} \\
& +i C\left(v_{n+1}+v_{n-1}-2 v_{n}\right) .
\end{aligned}
$$

Here $\gamma>0$ is the coefficient of the gain and loss acting on complex variables $u_{n}$ and $v_{n}$, which correspond, respectively, to the active and passive elements; real coefficient $\sigma$ accounts for the nonlinear frequency shift (the intrinsic nonlinearity of each element); $\kappa$ is the constant of the vertical coupling inside the dimer; and $C$ is a coefficient of the horizontal linear coupling between dimers in the chain [see Fig. 1(a)]. Using obvious transformations $\left(v_{n} \rightarrow-v_{n}\right.$, the complex conjugation, and staggering, respectively), one can fix $\kappa, C$, and $\sigma$ to be positive. Finally, by means of rescaling, we can set $\kappa=\sigma \equiv 1$, which leaves $\gamma>0$ and $C>0$ as irreducible parameters, the final form of the equations being

$$
\begin{aligned}
& \frac{d u_{n}}{d t}=\gamma u_{n}+i\left|u_{n}\right|^{2} u_{n}+i v_{n}+i C\left(u_{n+1}+u_{n-1}-2 u_{n}\right), \\
& \frac{d v_{n}}{d t}=-\gamma v_{n}+i\left|v_{n}\right|^{2} v_{n}+i u_{n}+i C\left(v_{n+1}+v_{n-1}-2 v_{n}\right) .
\end{aligned}
$$

First, we will derive approximate solutions for moving broad solitons, whose width is large in comparison with the lattice spacing, and study their almost elastic collisions, using a continuum counterpart of the discrete equations. We will also consider the case of narrow solitons, finding such strongly localized solutions numerically, starting from the anticontinuum (AC) limit. It will be demonstrated that the solitons with the amplitude exceeding a certain critical value become unstable. Because the instability is induced by the interplay of the gain and loss in the $\mathcal{P} \mathcal{T}$-symmetric system, the instability threshold naturally lowers with the increase of the strength of the gain and loss.

\section{SOLITONS}

\section{A. Analytical approximations}

We start with the analysis of the continuum limit $(C \rightarrow \infty)$ of Eq. (2), which leads to the following system of partial differential equations:

$$
\begin{aligned}
& \frac{\partial u}{\partial t}=+\gamma u+i|u|^{2} u+i v+i \frac{\partial^{2} u}{\partial x^{2}}, \\
& \frac{\partial v}{\partial t}=-\gamma v+i|v|^{2} v+i u+i \frac{\partial^{2} v}{\partial x^{2}},
\end{aligned}
$$

which possess exact soliton solutions:

$\left\{\begin{array}{c}u(t, x) \\ v(t, x)\end{array}\right\}=A \operatorname{sech}\left[\frac{A}{\sqrt{2}}(x-V t)\right] e^{i(V x / 2-\omega t)}\left\{\begin{array}{c}e^{i \delta / 2} \\ e^{-i \delta / 2}\end{array}\right\}$.

Here $A, A^{-1}, V, \omega$, and $\delta$ represent the amplitude, width, velocity, frequency, and intrinsic phase shift of the soliton:

$$
\begin{aligned}
\omega & =-A^{2}-\cos \delta+V^{2} / 4+A^{2} / 2, \quad \sin \delta=-\gamma, \\
\cos \delta & = \pm \Gamma, \quad \Gamma \equiv \sqrt{1-\gamma^{2}} .
\end{aligned}
$$

In the following, the case of $\cos \delta=\Gamma$ will be considered. Note that $\Gamma \leqslant 1$, and this solution exists for $\gamma \leqslant 1$. In the limit of the vanishing gain and loss, $\gamma \rightarrow 0$, Eqs. (3) become tantamount to the well-known model of dual-core nonlinear optical fibers. In that case, soliton (4) with $\cos \delta=\Gamma \equiv+1$ reduces to the symmetric soliton in the dual-core fiber, which loses its stability through the symmetry-breaking bifurcation at a finite value of the energy [17]. For the comparison with the discrete system introduced in the present work, it is important to mention that a similar symmetry-breaking instability of twocomponent solitons in the discrete counterpart of the dual-corefiber model (in fact, this discrete system may also be readily implemented in optics) was found in Ref. [18].

Although Eqs. (3) are dissipative, they are Galilean invariant; hence the soliton may move at an arbitrary velocity $V$. It is also worthy to note that the amplitude of the soliton may be arbitrary; i.e., the present solutions form a continuous family, unlike formally similar approximate $[11,13,15]$ and exact [12] 
soliton solutions previously found in the above-mentioned models of dual-core systems with the gain applied in one core and loss acting in the other. Those models were considered without the condition of the balance between the gain and loss in the general case (although the case of equal gain and loss had a special purport in that context too); therefore, they support solitons with two discrete values of the amplitude, one stable and one unstable.

For sufficiently wide solitons, i.e., for $A \ll \sqrt{C}$, the following approximate solution to the discrete Eq. (2) can be deduced from the continuum solution (4):

$$
\begin{aligned}
\left\{\begin{array}{l}
u_{n}(t) \\
v_{n}(t)
\end{array}\right\}= & A \operatorname{sech}\left[\frac{A}{\sqrt{2}}\left(\frac{n-x_{0}}{\sqrt{C}}-V t\right)\right]\left\{\begin{array}{c}
e^{i \delta / 2} \\
e^{-i \delta / 2}
\end{array}\right\} \\
& \times \exp \left\{i\left[\left(\frac{A^{2}}{2}+\Gamma-\frac{V^{2}}{4}\right) t+\frac{V\left(n-x_{0}\right)}{2 \sqrt{C}}\right]\right\},
\end{aligned}
$$

where $x_{0}$ is shift along the lattice. An example of the soliton propagation, generated by simulations of Eq. (2) with the initial conditions taken as per approximation (6), is displayed in Fig. 1(b). In this case, $A / \sqrt{2 C}=0.37$, i.e., the soliton is not very wide; nevertheless, approximation (6) yields good initial conditions for the soliton. The moving soliton practically does not radiate energy in the form of small-amplitude waves. On the contrary, Fig. 1(c) demonstrates that the soliton with a large velocity loses energy through the emission of radiation.

For narrow solitons, with $A \gtrsim \sqrt{C}$, the approximation based on the continuum limit is obviously irrelevant. In this case, solitons can be constructed numerically, starting from solutions which are exact ones in the anticontinuum limit, that correspond to $C=0$ in Eq. (2):

$$
\begin{aligned}
& \left\{\begin{array}{c}
u_{0} \\
v_{0}
\end{array}\right\}=A\left\{\begin{array}{c}
e^{i \delta / 2} \\
e^{-i \delta / 2}
\end{array}\right\} e^{-i \omega t}, \\
& u_{n}=v_{n}=0 \text { for } n \neq 0,
\end{aligned}
$$

where $A$ is an arbitrary amplitude, while the frequency and phase shift between the two components are given by

$$
\omega=-A^{2}-\cos \delta, \quad \sin \delta=-\gamma, \quad \cos \delta= \pm \Gamma .
$$

In the following the case $\cos \delta=\Gamma$ will be considered, following the choice adopted above for the broad solitons.

It follows from Eq. (8) that this solution does not exist at

$$
\gamma>1 \text { or } \omega<-\Gamma \text {. }
$$

On the other hand, for $C \neq 0$, one has the continuous-wave (CW) solution to Eq. (2), i.e., the wave with a constant amplitude, in the form of

$$
u_{n}=u_{0}, \quad v_{n}=v_{0},
$$

for all $n$, where $u_{0}$ and $v_{0}$ are defined by Eqs. (7) and (8).

We stress that, as well as the exact soliton solutions (4) and approximate solutions (6), the $\mathrm{AC}$ and $\mathrm{CW}$ solutions, given by Eqs. (7) and (10), respectively, form continuous families in spite of the fact that they exist in the dissipative system. This is a fundamental manifestation of the $\mathcal{P} \mathcal{T}$ symmetry in the present system.

\section{B. Stability analysis}

The next step of the analysis is the consideration of the stability of the AC solution (7) and $\mathrm{CW}$ solution given by Eq. (10). In the latter case, the perturbed CW state is looked for as

$$
\left\{\begin{array}{c}
\tilde{u}_{n} \\
\tilde{v}_{n}
\end{array}\right\}=\left\{\begin{array}{c}
\left(A+\epsilon_{1} e^{\alpha t-i k n}\right) e^{i \delta / 2} \\
\left(A+\epsilon_{2} e^{\alpha t-i k n}\right) e^{-i \delta / 2}
\end{array}\right\} e^{-i \omega t},
$$

where $\epsilon_{1}$ and $\epsilon_{2}$ are complex amplitudes of infinitesimal perturbations with eigenvalue $\alpha$ and wave number $k$. Substituting this expression into Eq. (2) and performing the linearization with respect to the perturbations yields four branches of the dispersion relation,

$$
\begin{aligned}
\alpha_{1,2} & = \pm 2 \sqrt{C(1-\cos k)\left[A^{2}-C(1-\cos k)\right]} \\
& \equiv \pm 2 \sqrt{\chi}, \\
\alpha_{3,4} & = \pm 2 \sqrt{\chi+\Gamma\left[-\Gamma+A^{2}-2 C(1-\cos k)\right]} .
\end{aligned}
$$

The CW solution is stable if condition $\operatorname{Re}\{\alpha\} \leqslant 0$ holds for all four eigenvalues at all $k$. For $C \neq 0$ it is sufficient to analyze this condition for $\alpha_{3,4}$, as inequality $\operatorname{Re}\left\{\alpha_{1,2}\right\} \leqslant 0$ follows from $\operatorname{Re}\left\{\alpha_{3,4}\right\} \leqslant 0$. The subsequent consideration demonstrates that the $\mathrm{CW}$ solution might be stable only if condition

$$
A^{2} \leqslant C(1-\cos k)
$$

holds for all $k$, which is impossible at $A \neq 0$. Thus, for $C \neq 0$, all the CW solutions (10) are modulationally unstable. This result also suggests that dark solitons are unstable in the dimer chain, because the corresponding $\mathrm{CW}$ background cannot be stable.

However, in the case of periodic boundary conditions, rather than the infinite chain, the $\mathrm{CW}$ state can be stable for $C \neq$ 0 if the ring-shaped chain is sufficiently short. As follows from Eq. (14), unstable are the modulational perturbations with wavelengths

$$
\lambda \equiv \frac{2 \pi}{k}>\frac{2 \pi}{\arccos \left(1-A^{2} / C\right)} .
$$

Thus, if the number of dimers in the chain with periodic boundary conditions satisfies condition

$$
N \leqslant \frac{2 \pi}{\arccos \left(1-A^{2} / C\right)},
$$

which makes the length of the chain smaller than the wavelength of the shortest unstable perturbation, the $\mathrm{CW}$ solution (10) is stable. Because $N$ cannot be smaller than 2 , the constraint

$$
A^{2} \leqslant 2 C
$$

follows from Eq. (15).

The stability analysis for the AC solution Eq. (7) can be performed by substituting Eq. (11) into Eq. (2) with $C=0$. In this case, Eqs. (12) and (13) reduce to $\alpha_{1,2}=0$ and $\alpha_{3,4}=$ $\pm 2 \sqrt{\Gamma\left(-\Gamma+A^{2}\right)}$, from which it follows that the AC solution is stable for

$$
A^{2} \leqslant\left(A_{\max }^{(0)}\right)^{2} \equiv \Gamma .
$$

These solutions, stable in the AC limit, are used below to construct numerical solutions for narrow solitons. 


\section{NUMERICAL RESULTS}

Discrete evolution equations (2) were integrated numerically, using a modification of an implicit, unconditionally stable Crank-Nicholson scheme with accuracy $\sim \tau^{4}$, where the time step was taken as $\tau=2 \times 10^{-5}$. Zero boundary conditions were employed, except for Sec. IV A, where the periodic boundary conditions were used.

\section{A. Unstable CW dynamics in the chain with periodic boundary conditions}

Interesting dynamics was observed in the chain with periodic boundary conditions for the initial conditions taken as per CW solution (10), in the case when the chain's length $N$ is only slightly greater than the critical value specified by Eq. (15), while conditions (16) and (17) are satisfied.

The instability of the system results in periodic appearance and disappearance of weakly localized states. This dynamics is illustrated by Fig. 2 for $\gamma=0.5, A=0.3, C=0.3$, and $N=8$ (the corresponding CW solution is stable for $N \leqslant 7$ ). Figure 2(c) displays the time variation of the localization parameter defined as

$$
l=\left(\sum_{n=0}^{N-1}\left|u_{n}\right|\right)^{-2}\left(\sum_{n=0}^{N-1}\left|u_{n}\right|^{2}\right) .
$$
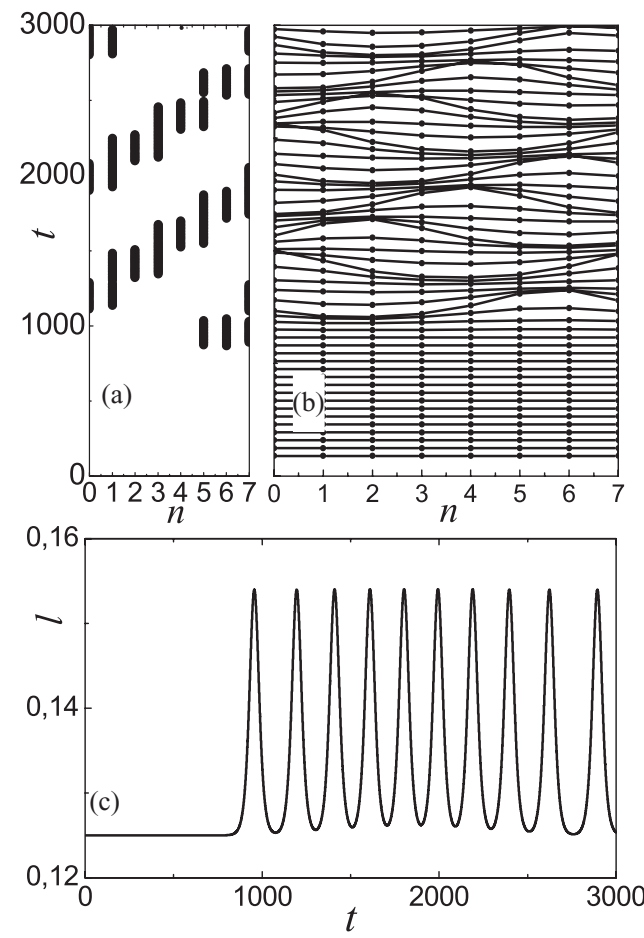

FIG. 2. Unstable dynamics in a compact $(N=8)$ ring-shaped chain of dimers with initial conditions corresponding to the $\mathrm{CW}$ state (10) with amplitude $A=0.3$ and model's parameters $\gamma=0.5$ and $C=0.3$. (a) Sites (dimers) in the $(n, t)$ plane with intensity greater than $1.1 A^{2}$ are shown in black, while others are not shown. (b) Evolution of intensity $\left|u_{n}(t)\right|^{2}$ [the picture for $\left|v_{n}(t)\right|^{2}$ is identical to this one, within the numerical accuracy]. (c) Evolution of the localization parameter $l$, defined as per Eq. (18).
Note that when $\left|u_{n}\right|=$ const, as in the unperturbed CW state, Eq. (18) yields $l=1 / N$. This is the minimal possible value, which tends to zero with the increase of $N$. On the other hand, when single $\left|u_{n}\right|$ is different from zero, the localization parameter attains its maximal value, $l=1$.

In the simulations, the only source of perturbations destabilizing the CW state (10) was rounding errors of the numerical code. In this case, the deviation of the solution from the initial unstable configuration becomes visible at $t \approx 900$. The unstable dynamics at $t>900$ results in quasi-periodic localizations and delocalizations of $\left|u_{n}\right|^{2}$, as seen in Fig. 2(c). The maximum of $\left|u_{n}\right|^{2}$ gradually moves along the chain with a nearly constant velocity, as seen in Figs. 2(a) and 2(b).

A similar effect of the periodic in time and space localization of the unstable $\mathrm{CW}$ solution, subject to periodic boundary conditions, was observed in the model of a diatomic chain of atoms [19]. A simple analytical model describing this effects was proposed in Ref. [20].

\section{B. Strongly localized onsite solitons}

Sharp (tightly localized) onsite solitons were constructed starting from the AC-limit solution (7), which is valid for $C=0$. After setting the initial conditions, the coupling constant was increased linearly in time from $C=0$ at $t=0$ to $C=0.15$ at $t=400$ and then kept constant. The total duration of the numerical run was $t=1000$. With such a slow increase of $C$, nearly stationary solitons were readily created (details of the stationary shape of the solitons are given below). The initial amplitude of the soliton in the AC limit was $A=0.6$. This amplitude satisfies the AC stability condition (17) for three considered values of the gain/loss parameter, $\gamma=0,0.3$, and 0.9 . The chain was composed of 21 dimers, which was sufficient for constructing tightly localized solitons.

Results of the simulations can be summarized as follows:

(i) The loss-gain coefficient $\gamma$ does not affect the profile and amplitude of the soliton, $\left|u_{n}\right|^{2}$ and $\left|v_{n}\right|^{2}$. This can be seen in Fig. 3, where the shapes of the solitons constructed for $\gamma=$ $0,0.3$, and 0.9 practically overlap. Coefficient $\gamma$ only affects the phase difference between complex quantities $u_{n}$ and $v_{n}$ and the frequency of the soliton.

The fact that the profiles of stationary solitons do not depend on $\gamma$ can be explained by noting that if $y_{n}=W_{n} e^{-i \omega t}$

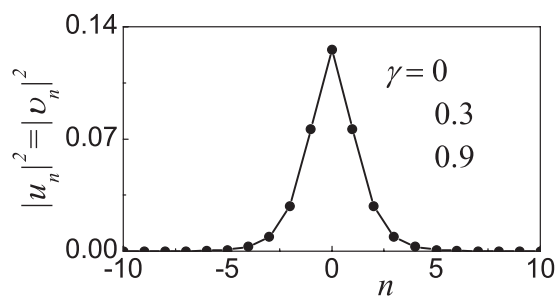

FIG. 3. Soliton profiles obtained from the anticontinuum limit by the slow increase of coupling constant $C$ at three different values of the gain-loss parameter, $\gamma=0,0.3$, and 0.9. Intensities $\left|u_{n}\right|^{2}$ and $\left|v_{n}\right|^{2}$ do not depend on $\gamma$, while the soliton's frequency and phase shift between $u_{n}$ and $v_{n}$ depend on $\gamma$, as explained in the text. 
is a stationary solution of the standard discrete nonlinear Schrödinger (DNLS) equation

$$
\frac{d y_{n}}{d t}=-i y_{n}+i\left|y_{n}\right|^{2} y_{n}+i C\left(y_{n+1}+y_{n-1}-2 y_{n}\right)
$$

then a stationary solution of Eq. (2) can be written as

$$
\left\{\begin{array}{c}
u_{n} \\
v_{n}
\end{array}\right\}=W_{n} e^{-i \omega t} e^{i t\left(1-\sqrt{1-\gamma^{2}}\right)}\left\{\begin{array}{c}
e^{i \delta / 2} \\
e^{-i \delta / 2}
\end{array}\right\},
$$

where $\delta$ is defined by Eq. (8). It is clear that solution (20) with $\gamma \neq 0$ differs from a stationary solution to Eq. (19), with $\gamma=0$, only by the frequency and phase shift, while their intensities are same. Thus, the discrete system based on Eq. (2), although being dissipative, supports the continuous family of solitons, being in that respect similar to the conservative DNLS equation, and different from generic discrete dissipative systems, such as the discrete complex Ginzburg-Landau equation [21].

(ii) To check that the numerically found solitons are stationary ones, we calculated the measure of nonstationarity at site $n, S_{n} \equiv d\left(\left|u_{n}\right|^{2}\right) / d t$, for three solitons constructed from the AC limit at $\gamma=0,0.3$, and 0.9. We found that the result does not depend on $\gamma$, as expected in view of the above comment. In the considered examples, the nonstationarity of the constructed solitons is weak $\left(\max \left\{S_{n}\right\}\right.$ does not exceed $0.0003)$, and it gets weaker for the solitons constructed with the use of $C$ increasing still slower in time.

(iii) The tightly pinned onsite-centered soliton remains stable if its amplitude is not too large to break the $\mathcal{P} \mathcal{T}$ symmetry. This conclusion is justified by monitoring the longterm dynamics of the discrete solitons constructed according to the protocol outlined above.

\section{Instability of intersite and twisted tightly localized solitons}

In contrast to the stable onsite-centered discrete solitons, outlined above, the analysis has revealed that all the intersitecentered solitons are unstable. We attempted to construct such solitons by starting, in the AC limit, with two adjacent excited sites, $n=0$ and 1, using Eq. (7) with $A=0.6$ as initial conditions, gradually increasing $C$ from $C=0$ at $t=0$ to $C=0.15$ at $t=400$, and keeping then $C$ constant.

In Fig. 4 we present the evolution of the intersite mode in the course of the increase of $C$. This mode is stable only in the absence of the coupling, $C=0$. At $C \neq 0$, one site spontaneously sucks the energy from the other, which ends with the establishment of a stable onsite-centered soliton. Note
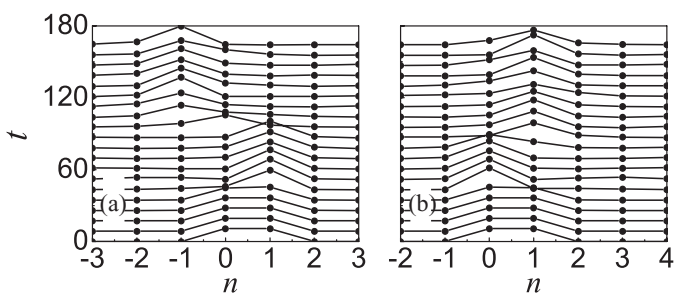

FIG. 4. Evolution of the intersite-centered soliton, grown from the AC initial conditions, following the increase of $C$ at two different values of $\gamma$. Eventually, one site spontaneously sucks the energy from the other one. The parameters are $A=0.6, C=0.15$, and $N=16$. (a) $\gamma=0.2$. (b) $\gamma=0.6$. that in the examples presented in Fig. 4 this happens at $t \approx 50$, i.e., before $C$ reaches the target constant value of 0.15 . We thus conclude that the intersite soliton is unstable. This result seems very plausible in view of the above-mentioned relation of stationary solitons in this system to those in the conventional DNLS equation (19), where all the intersite-centered solitons are unstable too [22].

We also attempted to excite twisted (antisymmetric) solitons, by starting, in the AC limit, with two adjacent excited sites, at $n=0$ and 1 , using Eq. (7) with $A=0.6$ and introducing the phase shift of $\pi$ between the sites. This mode too turns out to be unstable at $C>0$. In the usual lattices of the DNLS type, twisted solitons usually exist at large amplitudes [22], but the soliton's amplitude in the present $\mathcal{P} \mathcal{T}$-symmetric chain is limited by condition (17), which is a possible reason of the failure in looking for stable twisted solitons.

\section{Instability due to the $\mathcal{P} \mathcal{T}$-symmetry breaking}

According to the stability condition for the soliton in the AC limit, given by Eq. (17), increase in $\gamma$ results in a decrease of the critical value of the amplitude, $A_{\max }^{(0)}$, above which the localized mode becomes unstable. For the solitons in the chain with $C>0$, the corresponding critical value was determined numerically. To this end, we took, as the initial condition, a soliton constructed as described above (using the slow increase of coupling constant $C$ ), and then switched on a weak net pump of the energy into the soliton, by introducing a small mismatch $\epsilon$ in the gain/loss balance. In this case, Eq. (2) assumes the following form:

$$
\begin{aligned}
i \frac{d u_{n}}{d t}= & +(\gamma+\epsilon) u_{n}+i\left|u_{n}\right|^{2} u_{n} \\
& +i C\left(u_{n+1}+u_{n-1}-2 u_{n}\right)+i v_{n}, \\
i \frac{d v_{n}}{d t}= & -(\gamma-\epsilon) v_{n}+i\left|v_{n}\right|^{2} v_{n} \\
& +i C\left(v_{n+1}+v_{n-1}-2 v_{n}\right)+i u_{n} .
\end{aligned}
$$

In the stable regime, the field intensities in all dimers remain equal for the active and passive elements, both slowly increasing due to small excess gain, $\epsilon>0$. The system becomes unstable when the soliton's amplitude attains some critical value, $A_{\max }$. An example of the unstable behavior of the central dimer $(n=0)$ is shown in Fig. 5. In the unstable regime, the intensity in the active element sharply increases, while in its passive counterpart the intensity decays.

In Fig. 6 we compare the dependence of $A_{\max }^{2}$ on $\gamma$, as found numerically in this way, to the analytical result for the local mode in the AC limit, $\left(A_{\max }^{(0)}\right)^{2}$, as defined by Eq. (17). It is seen that they are in a good agreement, despite the fact that $A_{\max }^{2}$ pertains to the chain with $C \neq 0$. The numerical value is somewhat higher than the analytical estimate, which may be explained by a particular criterion of the instability adopted in the numerical study: the soliton was assumed to become unstable when the largest (over $n$ ) relative difference between $\left|u_{n}\right|^{2}$ and $\left|v_{n}\right|^{2}$ attained the level of $\max _{n}\left[\left.|| u_{n}\right|^{2}-\right.$ $\left.\left|v_{n}\right|^{2} \mid /\left(\left|u_{n}\right|^{2}+\left|v_{n}\right|^{2}\right)\right]>0.01$.

Results presented in Figs. 5 and 6 were obtained for the interdimer coupling constant $C=0.15$. Similar results were 


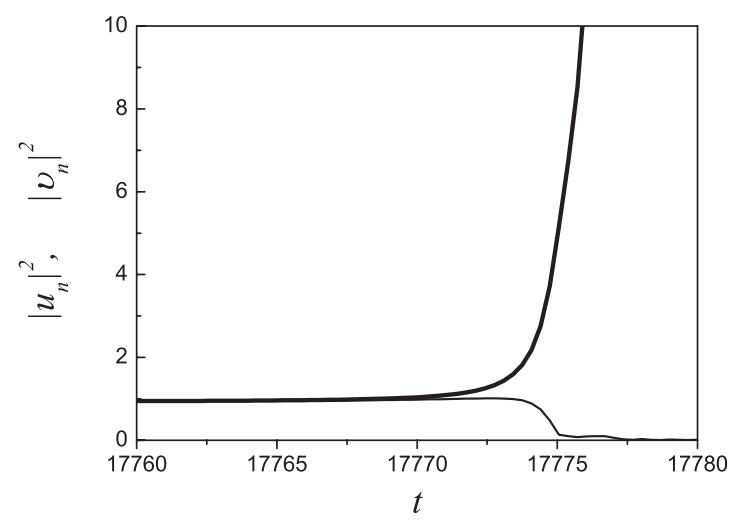

FIG. 5. Intensities of the fields at the central dimer, $n=0$, as functions of time, for the soliton in the chain of dimers with a small gain-loss mismatch: $\epsilon=10^{-5}, \gamma=0.4$, and $C=0.15$. Thick and thin lines pertain, respectively, to the elements with gain and loss, i.e., $\left|u_{n}\right|^{2}$ and $\left|v_{n}\right|^{2}$. The abrupt growth of $\left|u_{n}\right|^{2}$ signalizes the onset of the instability.

obtained for other values, up to $C=0.5$. In all the cases considered, the numerically found instability threshold $A_{\max }^{2}$ was very close to $\left(A_{\max }^{(0)}\right)^{2}$ predicted by Eq. (17) for the AC-limit soliton. Thus, the threshold practically does not depend on the coupling constant $C$. This result demonstrates a drastic difference in the dynamical properties of the discrete solitons in the $\mathcal{P} \mathcal{T}$-symmetric chain from its counterparts in the usual DNLS equation, where the instability of various soliton modes is solely determined by $C$ [22].

The dynamical blowup of unstable solitons is displayed in Fig. 7. Initial conditions were set using approximation (6) with $x_{0}=0$. In this case, $A^{2}=0.81>\Gamma=\sqrt{1-\gamma^{2}} \approx 0.436$; hence the AC stability condition Eq. (17) is violated. The result displayed in Fig. 7(c) suggests that, for $t<40$, the difference $\left.\Delta \equiv|| u_{0}(t)\right|^{2}-\left|v_{0}(t)\right|^{2} \mid$ increases with time exponentially, and then it starts to grow still faster. This change in the unstable dynamics is due to a qualitative change in the soliton's profile, that can be seen in Figs. 7(a) and 7(b). At $t<40$, intensities

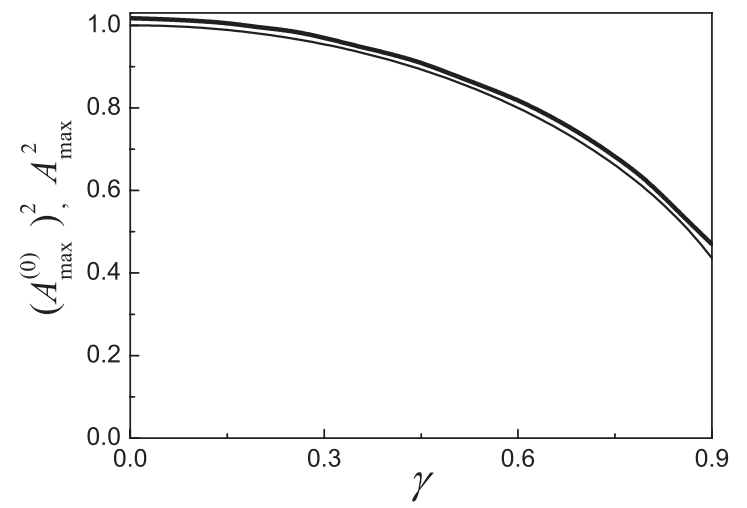

FIG. 6. Critical amplitude $A_{\max }^{2}$, above which the soliton's instability sets in, as a function of the loss-gain strength $\gamma$. The thick line is the numerical result for the solitons at $C=0.15$. The thin line represents the critical amplitude $\left(A_{\max }^{(0)}\right)^{2}=\Gamma$, as predicted by the stability condition (17) for the soliton in the anticontinuum limit $(C=0)$.
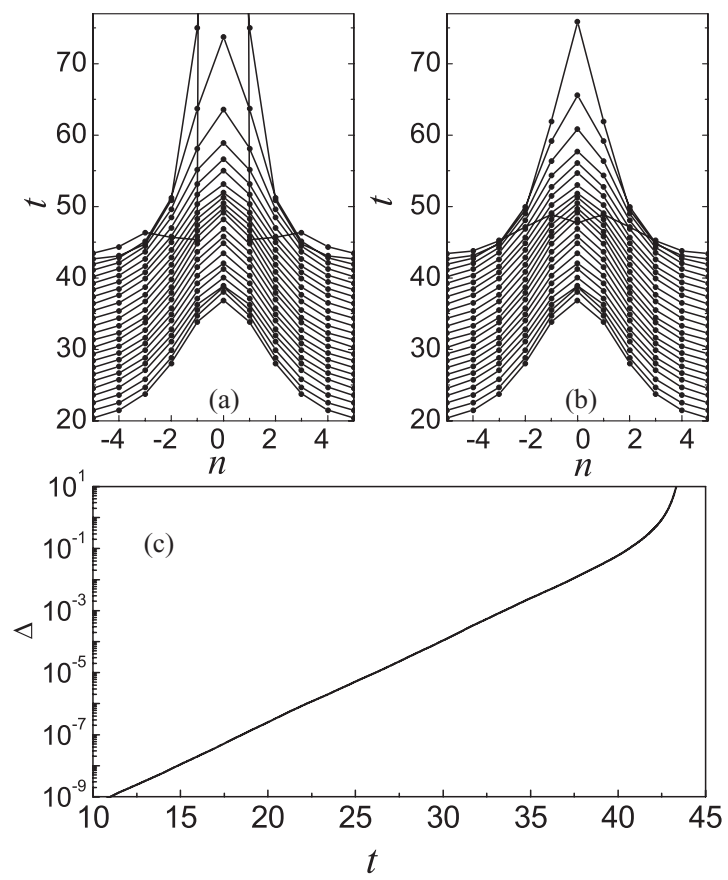

FIG. 7. Soliton dynamics in the unstable regime. Panels (a) and (b) display $\left|u_{n}(t)\right|^{2}$ and $\left|v_{n}(t)\right|^{2}$, respectively. (c) Difference in the intensities between the active and passive elements at the central dimer $(n=0), \Delta=\left.|| u_{0}\right|^{2}-\left|v_{0}\right|^{2} \mid$, as a function of time. Parameters are $\gamma=0.9, C=2.3, A=0.9$, and $V=0$.

$\left|u_{0}\right|^{2}$ and $\left|v_{0}\right|^{2}$ grow with time, but, after the difference between them becomes sufficiently large, $\left|u_{0}\right|^{2}$ starts to grow faster, while $\left|v_{0}\right|^{2}$ begins to decrease.

Results presented in Fig. 7 were obtained for the quiescent soliton $(V=0)$. We have also studied the influence of the soliton's velocity, $0<V \leqslant 0.5$, on the onset and development of the instability. It was found that the growth rate of $\Delta$ in the unstable regime and the critical soliton amplitude $A_{\max }^{2}$ do not depend on $V$.

This instability can be explained by comparison with the system of two linearly coupled DNLS equations, where the symmetric discrete soliton is destabilized by the symmetrybreaking bifurcation [18]. It seems plausible that the spontaneous trend to the symmetry breaking, due to the selfattraction in each chain, explains the onset of the instability in the present setting. However, there is a drastic difference from the dual-core DNLS system, where the symmetry breaking replaces the original destabilized soliton by stable asymmetric ones; in the $\mathcal{P} \mathcal{T}$ system this is impossible, as asymmetric modes cannot maintain the balance between the gain and loss, and instead exhibit the blowup, as shown in Fig. 7. A similar mechanism explains a stability limit for solitons in the model based on continual equations (3) [23].

\section{E. Peierls-Nabarro potential}

The effect of the Peierls-Nabarro (PN) potential in the chain of dimers is demonstrated by simulations presented in Fig. 8, where the initial conditions were set by using Eq. (6) with $x_{0}=0$. The parameters were chosen so as to produce a 


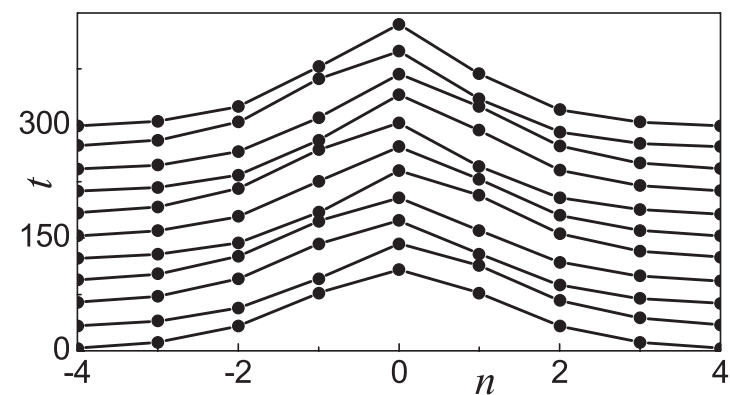

FIG. 8. Effect of the Peierls-Nabarro (PN) potential on the soliton dynamics. The initial momentum imparted to the soliton is not sufficient to overcome the PN potential barrier; therefore, the soliton oscillates near a local minimum of the potential. The parameters are $A=0.6, V=0.02, \gamma=0.5$, and $C=0.2$.

relatively sharp soliton when the PN barrier is noticeable. The soliton was kicked with a relatively small velocity $(V=0.02)$. The momentum given to the soliton was not sufficient to overcome the PN barrier; therefore, instead of propagating
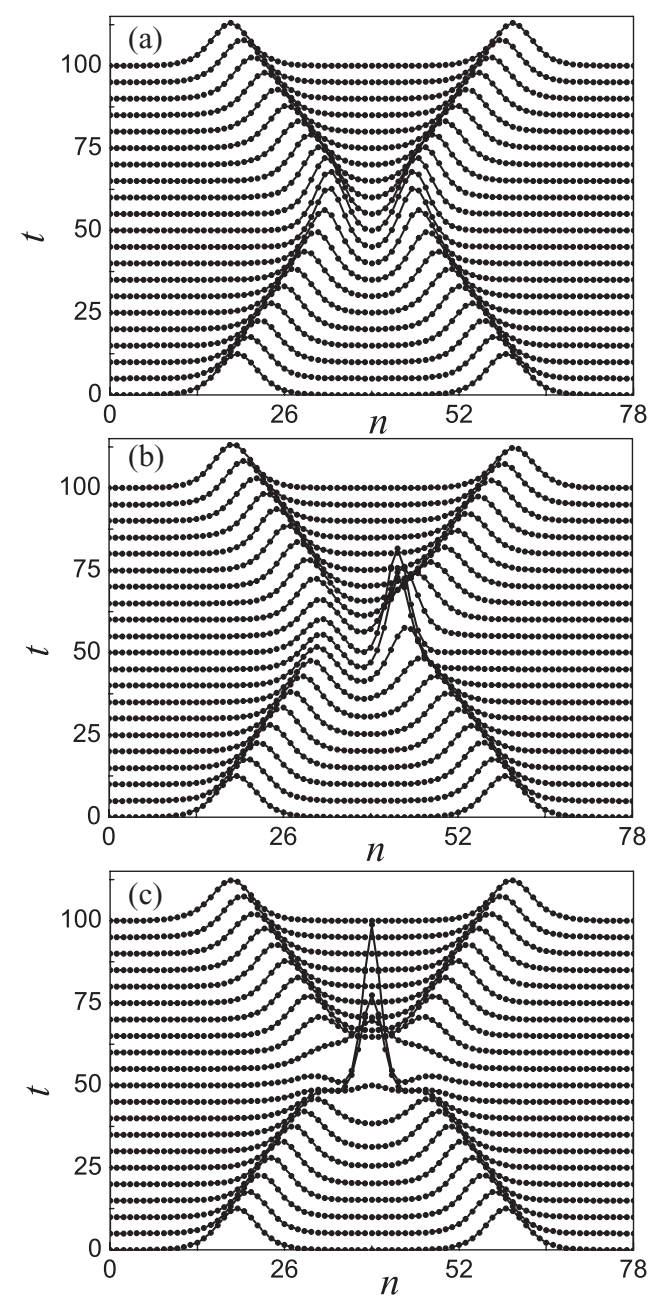

FIG. 9. Examples of quasielastic collisions between solitons for $\gamma=0.5, C=2, A=0.5$, and velocities $V= \pm 0.3$. The phase shift between the solitons in panels (a)-(c) is, respectively, $\pi, \pi / 2$, and 0 . along the chain, the soliton oscillates in a local well of the potential.

\section{F. Collisions between moving solitons}

The existence of moving solitons suggests a possibility to study collisions between them. Examples of collisions between identical solitons with opposite velocities $(V= \pm 0.3)$ are presented in Fig. 9. In this case, the solitons are relatively wide, hence the approximate solution (6) can be used for setting the initial conditions. The collisions are seen to be nearly elastic, with the solitons almost completely recovering their original shapes.

The solitons with phase shift $\pi$ in Fig. 9(a) repel each other, while the in-phase ones in Fig. 9(c) interact attractively. The collisions of the repelling out-of-phase solitons do not result in an overlap of their cores. For the phase shifts different from $\pi$, the overlap takes place; hence the largest intensity at the collision point is greater than in isolated solitons [see Fig. 9(b)], attaining the maximum for the in-phase collisions, as can be seen in Fig. 9(c). It is worthy to note that, although the instantaneous value of the amplitude at the collision point may be considerably larger than the critical amplitude defined by Eq. (17), this does not result in the onset of the instability, as the collision time is insufficient for that.

\section{CONCLUSIONS}

We have introduced a model describing an array of linearly coupled $\mathcal{P} \mathcal{T}$-symmetric dimers. Each dimer consists of linearly coupled active and passive elements with balanced gain and loss. The nonlinearity is represented by the conservative cubic term in the equation of motion for each element.

Using numerical simulations and analytical approximations, it was demonstrated that, in the regime of the sufficiently strong interchain coupling, the weakly discrete chain supports the free motion of solitary waves. At sufficiently small velocities, the moving solitary waves do not radiate energy and collide practically elastically, while solitons moving with large velocities emit radiation.

The chain of dimers was demonstrated to support tightly localized (sharp) stationary solitons in the regime of weak interdimer coupling (strong discreteness). Naturally, these strongly pinned solitons are immobile. A relation between the stationary solutions in the chain of dimers and the solutions to the standard discrete nonlinear Schrödinger equation was established.

Both wide and sharp solitons develop an instability if their amplitudes exceed the critical value, which leads to the blowup. This is a specific feature of the $\mathcal{P} \mathcal{T}$-symmetric chain, making the dynamical properties of the discrete solitons different from those in the usual DNLS system, in spite of the similarity in their shapes. An explanation to this feature, based on the trend to the spontaneous symmetry breaking, induced by the intrinsic self-attraction in the parallel chains, was proposed. The critical value decreases with the increase of the gain-loss parameter. Above the instability threshold, the $\mathcal{P} \mathcal{T}$-symmetry breaking occurs, resulting in the divergence of the intensity at the active element and decay of the intensity at the lossy one. A noteworthy finding is the independence of the critical 
amplitude on the lattice coupling constant and the soliton's velocity.

To continue the work in this direction, it would be interesting to study the chains consisting of more complex $\mathcal{P} \mathcal{T}$-symmetric elements (such as those with the nonlinearity of the gain and loss [10]) and to extend the analysis for two-dimensional lattices.

\section{ACKNOWLEDGMENTS}

B. A. Malomed and S. V. Dmitriev appreciate the hospitality of the Nonlinear Physics Center at the Australian National University. The work was partially supported by the Australian Research Council. S. V. Suchkov and S. V. Dmitriev acknowledge financial support from the Russian Foundation for Basic Research, grant 11-08-97057-p_povolzhie_a.
[1] C. M. Bender and S. Boettcher, Phys. Rev. Lett. 80, 5243 (1998); C. M. Bender, D. C. Brody, and H. F. Jones, ibid. 89, 270401 (2002); 92, 119902(E) (2004).

[2] C. M. Bender, Rep. Prog. Phys. 70, 947 (2007).

[3] F. Kh. Abdullaev, V. V. Konotop, M. Salerno, and A. V. Yulin, Phys. Rev. E 82, 056606 (2010); D. A. Zezyulin, V. V. Konotop, and G. L. Alfimov, ibid. 82, 056213 (2010).

[4] A. Ruschhaupt, F. Delgado, and J. G. Muga, J. Phys. A 38, L171 (2005); R. El-Ganainy, K. G. Makris, D. N. Christodoulides, and Z. H. Musslimani, Opt. Lett. 32, 2632 (2007).

[5] M. V. Berry, J. Phys. A 41, 244007 (2008); K. G. Makris, R. El-Ganainy, D. N. Christodoulides, and Z. H. Musslimani, Phys. Rev. Lett. 100, 103904 (2008); S. Klaiman, U. Günther, and N. Moiseyev, ibid. 101, 080402 (2008); S. Longhi, ibid. 103, 123601 (2009); Phys. Rev. A 82, 031801(R) (2010); Y. N. Joglekar, D. Scott, M. Babbey, and A. Saxena, ibid. 82, 030103(R) (2010).

[6] A. Guo, G. J. Salamo, D. Duchesne, R. Morandotti, M. VolatierRavat, V. Aimez, G. A. Siviloglou, and D. N. Christodoulides, Phys. Rev. Lett. 103, 093902 (2009); C. E. Ruter, K. G. Makris, R. El-Ganainy, D. N. Christodoulides, M. Segev, and D. Kip, Nature Physics 6, 192 (2010).

[7] Z. H. Musslimani, K. G. Makris, R. El-Ganainy, and D. N. Christodoulides, Phys. Rev. Lett. 100, 030402 (2008); H. Ramezani, T. Kottos, R. El-Ganainy, and D. N. Christodoulides, Phys. Rev. A 82, 043803 (2010); K. Li and P. G. Kevrekidis, e-print arXiv:1102.00809.

[8] A. A. Sukhorukov, Z. Xu, and Yu. S. Kivshar, Phys. Rev. A 82 043818 (2010).

[9] S. V. Dmitriev, A. A. Sukhorukov, and Y. S. Kivshar, Opt. Lett. 35, 2976 (2010).

[10] F. Kh. Abdullaev, Y. V. Kartashov, V. V. Konotop, and D. A. Zezyulin, Phys. Rev. A 83, 041805(R) (2011); A. E. Miroshnichenko, B. A. Malomed, and Y. S. Kivshar, ibid. 84, 012123 (2011).

[11] B. A. Malomed and H. G. Winful, Phys. Rev. E 53, 5365 (1996); J. Atai and B. A. Malomed, ibid. 54, 4371 (1996).
[12] J. Atai and B. A. Malomed, Phys. Lett. A 246, 412 (1998); H. Sakaguchi and B. A. Malomed, Physica D 147, 273 (2000).

[13] H. E. Nistazakis, D. J. Frantzeskakis, J. Atai, B. A. Malomed, N. Efremidis, and K. Hizanidis, Phys. Rev. E 65, 036605 (2002).

[14] P. V. Paulau, D. Gomila, T. Ackemann, N. A. Loiko, and W. J. Firth, Phys. Rev. E 78, 016212 (2008); P. V. Paulau, D. Gomila, P. Colet, N. A. Loiko, N. N. Rosanov, T. Ackemann, and W. J. Firth, Opt. Express 18, 8859 (2010); W. J. Firth and P. V. Paulau, Eur. Phys. J. D 59, 13 (2010); P. V. Paulau, D. Gomila, P. Colet, B. A. Malomed, and W. J. Firth, Phys. Rev. E 84, 036213 (2011).

[15] A. Marini, D. V. Skryabin, and B. A. Malomed, Opt. Express 19, 6616 (2011)

[16] B. A. Malomed, Chaos 17, 037117 (2007).

[17] E. M. Wright, G. I. Stegeman, and S. Wabnitz, Phys. Rev. A 40, 4455 (1989); A. I. Maimistov, Kvantovaya Elektron. (Moscow) 18, 758 (1991) [Sov. J. Quantum Electr. 21, 687 (1991)]; J. M. Soto-Crespo and N. Akhmediev, Phys. Rev. E 48, 4710 (1993); P. L. Chu, B. A. Malomed, and G. D. Peng, J. Opt. Soc. Am. B 10, 1379 (1993)

[18] G. Herring, P. G. Kevrekidis, B. A. Malomed, R. CarreteroGonzález, and D. J. Frantzeskakis, Phys. Rev. E 76, 066606 (2007).

[19] S. V. Dmitriev, A. A. Sukhorukov, A. I. Pshenichnyuk, L. Z. Khadeeva, A. M. Iskandarov, and Y. S. Kivshar, Phys. Rev. B 80, 094302 (2009)

[20] S. V. Dmitriev, A. A. Nazarov, A. I. Potekaev, A. I. Pshenichnyuk, and L. Z. Khadeeva, Russian Phys. J. 52, 132 (2009).

[21] N. K. Efremidis and D. N. Christodoulides, Phys. Rev. E 67, 026606 (2003); K. Maruno, A. Ankiewicz, and N. Akhmediev, Opt. Commun. 221, 199 (2003).

[22] P. G. Kevrekidis, The Discrete Nonlinear Schrödinger Equation: Mathematical Analysis, Numerical Computations, and Physical Perspectives (Springer, Berlin, 2009).

[23] R. Driben and B. A. Malomed, Opt. Lett., in press. 